Illinois State University

ISU ReD: Research and eData

Theses and Dissertations

4-10-2014

\title{
Current Trends In Concussion Management Among Athletic Trainers In Different Workplace Settings
}

Katelyn Persoage

Illinois State University, kpersoage@hotmail.com

Follow this and additional works at: https://ir.library.illinoisstate.edu/etd

Part of the Kinesiology Commons

\section{Recommended Citation}

Persoage, Katelyn, "Current Trends In Concussion Management Among Athletic Trainers In Different Workplace Settings" (2014). Theses and Dissertations. 123.

https://ir.library.illinoisstate.edu/etd/123

This Thesis is brought to you for free and open access by ISU ReD: Research and eData. It has been accepted for inclusion in Theses and Dissertations by an authorized administrator of ISU ReD: Research and eData. For more information, please contact ISUReD@ilstu.edu. 
CURRENT TRENDS IN CONCUSSION MANAGEMENT AMONG ATHLETIC TRAINERS IN DIFFERENT

WORKPLACE SETTINGS

Katelyn A. Persoage

48 Pages

May 2014

Context: There are many techniques used by athletic trainers in the management of concussions. Athletic trainers may use different management methods based on workplace setting but it is unclear what those differences may be. Objective: To determine the current concussion management protocols used by athletic trainers in different workplace settings.

Design: Quantitative analysis. Setting: Web-based survey. Patients or Other Participants: A link to an online survey was sent to 1000 Certified Athletic Trainers through the National Athletic Trainers' Association. One hundred twenty-seven participants responded to the survey from the following settings: College $(n=35)$, High School $(n=72)$, Non-Academic $(n=20)$. Main Outcome Measure(s): Data was collected describing the practices used by athletic trainers in the diagnosis and management of a concussion, as well as the return to play process following a concussive injury. Additional information described the involvement of a physician or sports medicine team throughout the process. Results: Participants reported managing $21.55 \pm 22.18$ concussions in an average year. High school athletic trainers diagnosed $84.7 \%$ of all concussions and made return to play decisions $72.2 \%$ of the time. The ImPACT test was used most often in this setting. A total of $88.6 \%$ of college athletic trainers reported having an assigned physician at their current workplace. A physician or sports medicine team was typically responsible for diagnosing 
concussions and making return to play decisions. Athletic trainers in non-academic settings refer to physicians for concussion diagnoses and return to play decisions. Conclusions: This study identified a number of differences in concussion practices among athletic trainers in different workplaces settings. In addition, the presence and involvement of a physician varied a considerable amount in the different settings. High school athletic trainers are primarily responsible for diagnosing and managing concussions more often than athletic trainers in the college and non-academic settings. Key Words: concussion management, return to play guidelines 
CURRENT TRENDS IN CONCUSSION MANAGEMENT AMONG ATHLETIC TRAINERS IN DIFFERENT WORKPLACE SETTINGS

KATELYN A. PERSOAGE

A Thesis Submitted in Partial Fulfillment of the Requirements

for the Degree of

MASTER OF SCIENCE

School of Kinesiology and Recreation

ILLINOIS STATE UNIVERSITY 
(C) 2014 Katelyn A. Persoage 
CURRENT TRENDS IN CONCUSSION MANAGEMENT AMONG ATHLETIC TRAINERS IN DIFFERENT WORKPLACE SETTINGS

KATELYN A. PERSOAGE

COMMITTEE MEMBERS:

Noelle Selkow, Chair

Peter Smith 


\section{ACKNOWLEDGMENTS}

I would like to thank my committee, Drs. Noelle Selkow and Peter Smith, for their hard work and dedication to this research project. Their guidance throughout this process was extremely valuable. I would also like to thank my family and friends for their continued support.

K. A. P. 


\section{CONTENTS}

CHAPTER

I. CURRENT TRENDS IN CONCUSSION MANAGEMENT AMONG ATHLETIC TRAINERS IN DIFFERENT WORKPLACE SETTINGS

Introduction

Methods

Participants

Instruments

Procedures

Statistical Analysis

Results

Discussion

Conclusion

Anatomy of the Head

Anatomy of the Brain

Neurovascular Structures of the Brain

Definition of a Concussion

Pathophysiology of a Concussion

Complications Following a Concussion

Evaluation of a Concussion

Concussion Management 
CHAPTER I

CURRENT TRENDS IN CONCUSSION MANAGEMENT AMONG ATHLETIC TRAINERS IN

DIFFERENT WORKPLACE SETTINGS

\section{Introduction}

Concussions are complex pathophysiological processes affecting the brain that are caused by traumatic biomechanical forces. ${ }^{1-3}$ The decision to return an athlete to play after a concussion can be very difficult for the sports medicine professional. In the past, a generic approach was taken to the assessment, management, and treatment of concussions, where all concussions were handled equally regardless of symptom severity and loss of consciousness. ${ }^{4}$ However, the American Orthopaedic Society for Sports Medicine (AOSSM) developed a set of guidelines in the late 1990's which focused on a more individualized concussion management protocol. $^{4}$

Traditionally, a symptom checklist has been used as a guideline for return to play decision-making. Several protocols have been developed around this idea; however, relying solely on symptom reporting can be problematic as some athletes will underreport symptoms in order to return to play sooner. ${ }^{5}$ This has caused current trends to shift towards a multi-faceted approach when returning athletes to competition. This approach includes a graded symptom checklist, neuropsychological testing and balance assessment. ${ }^{3,6}$ 
Guidelines established at the 2001 International Conference on Concussion in Sport in Vienna advocate a stepwise return-to-play program with gradually increasing intensity, starting with complete rest and ending with a return to game play. ${ }^{1}$ These guidelines have remained constant throughout subsequent conferences. This program should be implemented once the athlete reports being symptom-free and neurocognitive tests return to baseline scores. ${ }^{1-3}$ The National Athletic Trainers' Association suggests a similar protocol. ${ }^{5,6}$

While athletic trainers often deal directly with concussed athletes, medical clearance by a physician may be required before the athlete can return to competition. High level sports teams often have an assigned physician who has been involved throughout the injury. However, many athletes will obtain clearance from a physician who has not been present throughout the post-concussion progression, and who may not be familiar with current concussion assessment and management practices.

Several studies ${ }^{7-9}$ have examined the current techniques used by athletic trainers in the assessment and management of concussions. The results of these studies have indicated that while athletic trainers are using a variety of methods to diagnose and manage concussions, they are still lacking in some areas. One study ${ }^{7}$ in 2005 found that $97 \%$ of respondents were not using all three suggested components of the multi-faceted approach in return to play decision making. Since 2005, the use of neuropsychological testing and the BESS test for balance have increased among athletic trainers. ${ }^{7,9}$

A 2013 study ${ }^{9}$ identified differences in concussion management and return to play decision making based on workplace setting. Athletic trainers in the high school setting typically used non-computerized neurocognitive testing and relied on return-to-play guidelines and 
physician recommendations for return to play decision-making. ${ }^{9}$ Conversely, athletic trainers in the college setting used neurocognitive testing, BESS, SCAT2 and symptom checklists to make return to play decisions. They were also more likely to make these decisions on their own rather than referring athletes to a physician. ${ }^{9}$

While these studies provide some insight into the techniques used by athletic trainers to appropriately manage a concussion, there is limited research to show which practices are being used in different workplace settings. Therefore, the purpose of this study is to determine the current concussion management protocols used by athletic trainers in different workplace settings for a safe and effective return to play. In addition, this study looked at physician involvement during the process. It is hypothesized that athletic trainers will be varied in their protocols based on their work environment

\section{Methods}

The design of the study was a quantitative analysis of the current trends in concussion management and return to play protocols among athletic trainers in different workplace settings.

\section{Participants}

Participants consisted of 1000 members randomly selected from the National Athletic Trainers' Association (NATA) database. All participants were Certified Athletic Trainers from a variety of work settings. Any participant who indicated that they were not currently practicing as an athletic trainer was excluded from the study.

Consent to participate in the study was implied by completing the survey. Participants were permitted to omit answering questions if they chose to do so. The Institutional Review Board approved the study. 
One hundred thirty-four athletic trainers completed the study. Seven respondents indicated that they were not currently practicing or were working in an administrative role and were excluded from the study, leaving a total of 127 responses. Respondents were grouped into three categories based on workplace setting: high school $(n=72)$, college $(n=35)$, and nonacademic $(n=20)$. College athletic trainers came from Division I, II and III athletics, club sports and fine arts programs. Athletic trainers from non-academic settings worked in private clinics, hospitals, law enforcement, military, government and industrial settings.

Respondents reported having an average of $11.4 \pm 8.6$ years of experience. The most common sports covered by respondents were football, baseball/softball, basketball, volleyball, soccer, wrestling, track and field, and lacrosse.

\section{Instruments}

An online survey was used to collect data. The survey was hosted by Select Survey and took approximately $10-15$ minutes to complete. The survey included general questions on demographics, number of years certified, job description and work setting. A second section focused on concussion education received during schooling and in continuing education courses, number of concussions seen per year at current job, typical concussion diagnostic tools used, concussion treatment used, and return to play considerations. In addition, several questions were used to determine the presence of a physician and the physician's involvement throughout the process. Participants were free to withdraw from the survey at any time and were not required to complete all questions. 


\section{Procedures}

An email with a link to the survey was sent to 1000 certified athletic trainers randomly generated through the NATA's survey service. Participants were given four weeks to return the survey and sent a reminder at the halfway point.

\section{Statistical Analysis}

Data was analyzed using Microsoft Excel Version 14.3.7 (Microsoft Corporation, 2010 and SPSS Version 20 (IBM, 2011, Chicago IL). A chi-square test of independence was used to determine associations among the data. The $\alpha$ level was set $a$ priori at .05 for all tests.

\section{Results}

Concussion Frequency

Overall, athletic trainers reported $21.55 \pm 22.18$ concussions per year. The average number of concussions per year was highest in the high school setting, with athletic trainers managing $27.52 \pm 22.98$ concussions per year. In the college setting, $11.90 \pm 12.67$ were observed each year, while the non-academic settings saw an average of $15.63 \pm 25.10$ concussions per year.

Concussion Diagnosis

Across all work settings, the athletic trainer was responsible for diagnosing a concussion $66.9 \%$ of the time, with a combined effort from the sports medicine team as the second most common answer selected $\left(\chi_{127}^{2}=36.922, p<0.001\right.$, Cramer's $\left.V=0.381\right)$. When separated into work setting, high school athletic trainers were twice as likely to be responsible for making this decision compared to athletic trainers in the college or non-academic setting, and diagnosed $84.7 \%$ of all concussions. College athletic trainers were responsible for diagnosing $45.7 \%$ of concussions, whereas a sports medicine team or team physician were equally as likely to 
diagnose a concussion and combined for $45.7 \%$ of all diagnoses. Similar results were seen in the non-academic setting, with athletic trainers diagnosing $40.0 \%$ of concussions and a sports medicine team or team physician diagnosing a combined total of $45.0 \%$ of all concussions.

A significant difference was seen in the use of the ImPACT test in different settings. A total of $62.2 \%$ of respondents used the ImPACT test as part of their concussion diagnosis $\left(\chi_{127}^{2}=9.751, p=0.008\right.$, Cramer's $\left.V=0.277\right)$. In the high school setting, the ImPACT test was used by $72.2 \%$ of respondents. College athletic trainers used ImPACT $57.1 \%$ of the time, while nonacademic athletic trainers used the test $35.0 \%$ of the time.

Concussion Management

Some differences were seen in the techniques used by athletic trainers to manage the symptoms of a concussion. Bed rest was used as a management strategy by $42.2 \%$ of athletic trainers in the study $\left(\chi_{127}^{2}=7.607, p=0.022\right.$, Cramer's $\left.V=0.245\right)$. When separated into work setting, bed rest was used more often in the high school setting $(52.8 \%)$ than in college $(25.7 \%)$ or non-academic (35.0\%) settings.

Activity modification was another strategy commonly used in the treatment of a concussion. A total of $81.9 \%$ of all respondents reported using activity modification $\left(\chi_{127}^{2}=8.590\right.$, $p=0.014$, Cramer's V $=0.260$ ). College athletic trainers were least likely to use activity modification as treatment for concussion symptoms, with only $65.7 \%$ of college athletic trainers using this strategy. Activity modification was used more often in other settings, with $87.5 \%$ of high school and $90.0 \%$ of non-academic athletic trainers selecting this response.

Return to Play Decision

Some differences existed between work settings as to who was responsible for deciding whether or not an athlete is ready to return to play. In the high school setting, $72.2 \%$ of athletic 
trainers reported that they were responsible for making this decision $\left(\chi_{127}^{2}=49.357, p<0.001\right.$, Cramer's V $=0.441$ ). In the college setting, the team physician was selected as the primary decision-maker by $34.3 \%$ of athletic trainers. An additional $28.6 \%$ selected sports medicine team, and $22.9 \%$ reported that the athletic trainer made this decision. In the non-academic settings, many different individuals made the return to play decision, including the athletic trainer, team physician, sports medicine team and a general practitioner.

A step-wise return to play process, which is regularly advocated at the International Conference on Concussion in Sport ${ }^{1-3}$, is used by many athletic trainers to progressively return an athlete to competition. A total of $70.1 \%$ of all athletic trainers in this study reported using this process $\left(\chi_{127}^{2}=7.245, p=0.027\right.$, Cramer's $\left.V=0.239\right)$. However, there are differences between workplace settings. High school athletic trainers (79.2\%) use this process more than those in college $(54.3 \%)$ or non-academic $(65.0 \%)$ settings.

Presence and Involvement of a Doctor

A chi-square test for independence indicated an association between workplace setting and the presence of an assigned doctor, $\chi 2(1, n=127)=11.639, p=0.003$, Cramer's V $=0.303$. Athletic Trainers working in a college setting reported having a doctor assigned to their workplace in $88.6 \%$ of cases, while those in high school or non-academic settings only had an assigned doctor in $55.65 \%$ and $60 \%$ of cases, respectively.

Of those who reported having an assigned doctor, $57.1 \%$ of collegiate Athletic Trainers listed a general practitioner or family doctor as the physician in charge of the care of their athletes. This is a significant difference from high school (20.8\%) and non-academic Athletic Trainers (25.0\%) $\left(\chi 2_{127}=14.855, p=0.001\right.$, Cramer's V=0.342). 
Participants who reported having an assigned doctor were asked to rate the doctor's involvement throughout the concussion process on a scale of $0-10$, with $0=$ not at all involved and $10=$ involved in every step of the process. Overall, athletic trainers reported $5.6 / 10$ on the scale. Doctors in the college $(6.6 / 10)$ and non-academic $(6.2 / 10)$ settings appeared to be more involved than those in the high school setting (4.7/10).

These same participants were also asked to rate their assigned doctor's ability to properly manage a concussion. A scale of 0-10 was used, with $0=$ not at all confident in doctor's ability and $10=e x t r e m e l y$ confident in doctor's ability. Overall, athletic trainers with an assigned doctor rated his or her ability at 7.8/10. Athletic trainers in the college setting were most confident with their doctor's concussion management skills, giving an average rating of 9.4/10. High school athletic trainers rated their doctors a 7.4/10, while those in non-academic settings only averaged a rating of $6.2 / 10$.

Concussion Preparedness

Participants were asked a series of questions regarding concussion preparedness. Each question was rated on a scale of $0-10$, with $0=$ not at all prepared and $10=$ extremely well prepared.

When asked if they felt that they received enough education on concussion management during post-secondary education to become an athletic trainer, participants reported an average of $6.9 / 10$. Athletic trainers rated themselves as $9.3 / 10$ when asked if they felt they were currently prepared to manage a concussion, should the situation arise. Over three-quarters of respondents $(78.7 \%)$ believe that their current work setting is adequately prepared to manage a concussion. No significant differences were seen between groups for these variables. 


\section{Discussion}

The data from this study indicates that there are several important differences in concussion practices of athletic trainers in different workplace settings. In most cases, the greatest difference was observed between college and non-academic settings. High school settings had similarities to both college and non-academic settings. Athletic trainers in high schools appear to have the most responsibility in diagnosing and managing a concussion, as well as deciding how and when to return an athlete to play. In the college and non-academic settings, input from physicians and sports medicine teams were more common.

There was a difference in concussion frequency compared to previous studies. A study from $1999^{8}$ reported an average of 7.0 concussion every year. That number rose to 8.2 in $2005^{7}$, 10.7 in $2013^{9}$ and 21.6 as a result from this study. The reason for this increase could be due to the continued prevalence of concussion information in the media and literature, as well as the fact that many respondents from this study work in contact sports.

In the high school setting, athletic trainers were nearly twice as likely to be the person responsible for diagnosing a concussion compared to college or non-academic settings. The ImPACT test was also used more often at the high school level than in other settings. This is in contrast to a 2013 study $^{9}$, in which college settings used computerized neuropsychological testing more often than high schools. Bed rest was used as a management strategy more often in high schools than any other setting. Most athletic trainers at this level also used activity modification in the management of a concussion. High school athletic trainers were relied on to make return to play decisions more often than those in college and non-academic settings, with $72.2 \%$ of high school athletic trainers reporting that they were the person in charge of this decision. A step-wise return to play protocol, where the athlete is gradually returned to 
competition through progressively intensified physical activity, was used in many high school settings. It is interesting to note that although the ImPACT test was used most at the high school level in diagnosis, there were no significant differences in settings with regards to the use of this test in the return to play process. The presence of a physician at the high school level was much less common than at the college level. If a physician was present, they appeared to be less involved throughout the process than a college-level physician. High school athletic trainers also seemed to be less confident in their physician's concussion management abilities.

At the college level, the concussion management process was somewhat different than that of a high school. Physicians were present in many more cases, and often were responsible for making diagnoses and return to play decisions. Athletic trainers worked in conjunction with a sports medicine team more often than the other settings. This finding is in contrast to that of a 2013 study $^{9}$, where athletic trainers at the college level made return to play decisions rather than referring the athlete to a physician. Athletic trainers in the college setting appeared to have more confidence in their assigned physician, and noted that the physician was more involved in the process than physicians in other settings. College athletic trainers used the ImPACT test less than high school athletic trainers. This finding may not be a true representation of current practices. Anecdotally, athletic trainers at the college level typically have larger budgets and access to more advanced methods of testing.

In non-academic settings, physicians were present much less than in college settings, though athletic trainers typically referred athletes for concussion diagnoses and return to play decisions. In cases where a physician was present, they were more involved than at the high school setting, though non-academic athletic trainers had the least amount of faith in their physicians' abilities. Only $35.0 \%$ of athletic trainers in this setting reported using ImPACT testing 
in the diagnostic process. However, this finding correlates with the fact that athletic trainers are typically not the ones to diagnose a concussion in non-academic workplaces. Athletic trainers in non-academic settings used activity modification to manage concussion symptoms more than those in any other setting.

Our findings indicate that athletic trainers in the high school setting have the most responsibility. They see the most concussions, and are responsible for diagnosis, management and return to play decisions often without the help of a doctor or sports medicine team. While they are using some updated testing methods, such as ImPACT, they may not have access to as many resources as a college or non-academic setting. These findings continue to emphasize the need for more standardized methods of concussion management among all athletic trainers. It is also crucial to work towards a team-based approach at the high school level. Athletic trainers must work in conjunction with other sports medicine professionals, especially physicians, in order to ensure that athletes are receiving the best possible care.

While there were no significant differences between workplaces, it is interesting to note that $28.35 \%(36 / 127)$ of athletic trainers reported feeling that they have returned an athlete to play too soon following a concussion. A number of reasons were given as to why these decisions were made. Many of these athletic trainers have had athletes hide symptoms in order to return to play sooner. This is a troubling fact and emphasizes the need for other methods of evaluation such as neurocognitive testing and balance assessments. Another response commonly listed was that athletes had been returned too early in the past before the creation and implementation of concussion guidelines. Many athletic trainers who reported this as a reason noted that this had occurred early on in their career, but has not happened for many years. In some cases, athletic trainers did not have an established protocol at their place of employment, reinforcing the need 
for standardized practices among all athletic trainers. Finally, many athletic trainers reported that their athlete had received clearance from a physician even though the athletic trainer had suggested that the athlete was not yet ready for competition. This is perhaps the most worrisome response. In order to provide the best care for the athlete, all involved medical personnel need to be adequately educated in concussion management. In addition, athletic trainers, physicians and other members of the sports medicine team need to work in conjunction to decide the best course of action for the athlete.

Athletic trainers appear to feel confident in both their own concussion management abilities and the current practices of their workplace. However, they rated their educational preparedness with regards to concussion management as only 6.9/10. This finding suggests that more time should be spent reviewing concussion practices during athletic training education.

This study was limited by the low response rate (13.4\%), as well as the inherent limitations of survey research. It was expected that all participants understood and answered questions honestly, however there is no way to confirm this. This study was also limited due to the inclusion of "other" as an option for many questions. While this allowed for a greater variety of answers, it may have had an effect on significance.

\section{Conclusion}

The findings of this study indicate that there are several important differences between athletic trainers in different workplace settings. High school athletic trainers appear to be most varied in their methods, exhibiting similarities to both college and non-academic settings. Continued progress towards a standardized set of guidelines will help to eliminate these differences among work settings. The lack of physician involvement in the high school and nonathletic settings reinforces the need for sports medicine professionals to work together to 
properly manage concussions. Future research should focus on identifying other trends in concussion management practices and the involvement of physicians and sports medicine teams in the concussion process. 
CHAPTER II

REVIEW OF RELATED LITERATURE

\section{Anatomy of the Head}

The brain is located within the skull, a collection of 8 cranial bones and 14 facial bones. ${ }^{10}$ The cranium is composed of the occipital bone, two parietal bones, the frontal bone, two temporal bones, the sphenoid and the ethmoid. The face is composed of two nasal bones, two maxillae, two lacrimal bones, two zygomatic bones, two palatine bones, two inferior nasal concha, the vomer bone and the mandible. ${ }^{10}$ With the exception of the mandible, these bones are fused together and immovable, providing a strong protective casing for the brain.

The occipital bone is located on the posterior-inferior portion of the cranium. It is trapezoidal in shape and convex. ${ }^{10}$ The foramen magnum is a large opening within the occipital bone that allows the passage of the brain stem, as well as various nerves, veins and arteries from the brain to the spinal cord..$^{10}$ It articulates with the left and right parietal bones at the lambdoid suture ${ }^{11}$, the left and right temporal bones laterally, and inferiorly with the sphenoid and $^{\text {atlas. }^{10}}$

The parietal bones are located on the superior-lateral portion of the cranium, and create the roof of the skull. ${ }^{10}$ They are quadrilateral in shape. They articulate with one another superiorly at the sagittal border. ${ }^{10}$ In addition, the parietal bones articulate with the frontal bone at the coronal suture ${ }^{11}$, the temporal bones inferiorly at the squamous suture ${ }^{11}$, the sphenoid 
anterior and inferior at the anterior squamous border, and the occipital bone at the lambdoid suture. $^{11}$

The frontal bone is located on the anterior superior portion of the cranium. There are two portions of this bone: the squama, which creates the forehead, and the orbital portion, which creates the roof of the orbital and nasal cavities. ${ }^{10}$ The squama articulates with the parietal bones at the coronal suture. ${ }^{11}$ The orbital portion articulates laterally with the sphenoid bone. $^{10}$

The temporal bones are located on the lateral aspect of the cranium. They are separated into five parts: the squama, the petrous, the mastoid, the tympanic part and the styloid process. ${ }^{10}$ The squama articulates with the squamous border of the parietal bone at the squamous suture ${ }^{11}$ and the sphenoid anteriorly. The mastoid portion articulates with the occipital bone at the occipital groove. ${ }^{10}$ Located within the temporal bone is the petrotympanic fissure, which leads into the tympanic cavity.

The sphenoid is located on the inferior portion of the skull. It is composed of a body, two great wings, two small wings, and two pterygoid processes. ${ }^{10}$ The body is hollow, which allows two sphenoidal sinuses to pass through. ${ }^{10}$ The sphenoid articulates with the vomer, ethmoid, frontal, occipital, parietal, temporal, zygomatic and palatine bones. ${ }^{10}$

The ethmoid bone is located on the anterior portion of the cranium between the orbits. It forms a portion of each orbit, as well as the roof of the nose. ${ }^{10}$

The nasal bones are located in the upper medial portion of the face. They form the bridge of the nose. They are different sizes and shapes in different individuals, resulting in a visible difference in nose shape. ${ }^{10}$ 
The maxilla are two large facial bones that form the upper portion of the jaw. ${ }^{10}$ They contribute to the formation of the roof of the mouth, the floor and lateral wall of the nose, and the floor of the orbit. ${ }^{10}$

The lacrimal bones are the smallest and weakest bones in the face. ${ }^{10}$ They are located in the anterior portion of the medial wall of the orbit. They articulate with the maxilla anteriorly and inferiorly, the ethmoid posteriorly, and the frontal bone superiorly. ${ }^{10}$

The zygomatic bones are located on the upper lateral portion of the face. They form the prominence of the cheek and the lateral wall and floor of the orbit. ${ }^{10}$ The zygomatic bones articulate with the frontal, sphenoidal, temporal and maxilla bones. ${ }^{10}$

The palatine bones are located in the posterior nasal cavity between the maxilla and the sphenoid. They form the floor and lateral wall of the nasal cavity, the roof of the mouth, as well as the floor of the orbit. ${ }^{10}$ They articulate with each other, as well as the sphenoid, ethmoid, maxilla, inferior nasal concha, and vomer bones. ${ }^{10}$

The inferior nasal concha are located along the lateral wall of the nasal cavity. ${ }^{10}$ They articulate with the ethmoid, maxilla, lacrimal and palatine bones. ${ }^{10}$

The vomer forms the posterior-inferior portion of the nasal septum. ${ }^{10}$ It articulates with the sphenoid and ethmoid bones, as well as both maxilla and palatine bones. In addition, the vomer articulates with the nasal cartilage. ${ }^{10}$

The mandible is the largest and strongest bone in the face. ${ }^{10}$ It is the only movable bone in the cranium. Its main purpose is to hold the lower teeth. ${ }^{10}$ The mandible articulates with the two temporal bones at the temporomandibular joint. ${ }^{10}$

There are several important bony landmarks on the skull. These typically occur at the junctions of two or more sutures. The pterion is located on the lateral aspect of the skull, at the 
junction between the greater wing of the sphenoid, frontal, temporal and parietal bones. Located beneath this landmark is the anterior division of the medial meningeal artery. ${ }^{11} \mathrm{~A}$ fracture of the pterion may result in a rupture of the medial meningeal artery, resulting in a lifethreatening emergency. The asterion is also located on the lateral skull at the junction between the parietomastoid, occipitomastoid and lambdoid sutures. ${ }^{11}$

On the posterior aspect of the skull, the inion is located at the most prominent portion of the external occipital protuberance. ${ }^{11}$ The lambda is located at the junction between the sagittal and lambdoid sutures. ${ }^{11}$

The vertex and bregma are both located on the superior aspect of the skull. The vertex forms the most superior point of the skull, and is located near the centre of the sagittal suture. ${ }^{11}$ The bregma is formed by the intersection of the sagittal and coronal sutures. ${ }^{11}$

The glabella is located at the most anterior portion of the forehead, superior to the nose. ${ }^{11}$ The nasion is located at the intersection of the frontonasal and internasal sutures. ${ }^{11}$

The scalp forms a protective covering for the cranium. It is composed of skin and subcutaneous tissue and stretches from the superior nuchal lines of the occipital bone to the supraorbital margin of the frontal bone. Laterally it extends down to the zygomatic arches. ${ }^{11}$

The scalp is composed of five layers. ${ }^{11}$ Layers one through three are interconnected and move as a single unit. The outermost layer of the scalp is composed of skin. It contains sweat glands sebaceous glands and hair follicles. It has many arteries, veins and lymphatic vessels. ${ }^{11}$ The second layer is formed by a thick layer of connective tissue containing many cutaneous nerves. ${ }^{11}$ The third layer of the scalp is the epicranial aponeurosis. This is a layer of tendinous material that forms an attachment for muscles of the forehead and occiput. ${ }^{11}$ The fourth layer of the scalp is composed of spongy areolar tissue. The function of the fourth layer is to allow the 
first three layers to move freely. The areolar tissue contains many spaces that may fill with fluid during injury or infection. ${ }^{11}$ The innermost layer of the scalp is the pericranium. It is a thick layer of connective tissue that is fixed to the underlying bone, forming the external portion of the periosteum. $^{11}$

\section{Anatomy of the Brain}

The brain is a large and important organ in the body. It is responsible for all actions, both voluntary and involuntary. It is composed of the cerebrum, cerebellum, diencephalon, and the brainstem. ${ }^{11}$

The cerebrum has many functions, including coordination of muscle action, sensory integration, memory, reasoning and emotion. ${ }^{12}$ The cerebrum is divided into left and right hemispheres by the longitudinal cerebral fissure ${ }^{11}$, and separated from the cerebellum by the transverse cerebral fissure. ${ }^{13}$ Each hemisphere has three main regions: superficial gray matter, internal white matter and basal nuclei located within the white matter. ${ }^{13}$ The hemispheres can also be divided into frontal, parietal, occipital, temporal and insula lobes. ${ }^{11}$

The gray matter, or cerebral cortex, is where conscious thought occurs. This includes actions such as sensation, communication, memory, understanding and voluntary movement. ${ }^{13}$ The gray matter contains neuron cell bodies, dendrites, glia and blood vessels. It is $2-4 \mathrm{~mm}$ thick, and approximately $40 \%$ of total brain mass. ${ }^{13}$ Each hemisphere of the cerebral cortex contains motor, sensory and association areas responsible for actions on the opposite side of the body. While both hemispheres are active with most activities, each hemisphere tends to dominate certain tasks. The left hemisphere is typically responsible for language, math and logic, while the right hemisphere is used in visual-spatial tasks, emotion and intuition. ${ }^{13}$ 
The motor areas are located within the posterior aspect of the frontal lobe and include the primary motor cortex, the premotor cortex, Broca's area and the frontal eye field. ${ }^{13}$ The primary motor cortex is located in the precentral gyrus of the frontal lobe..$^{13}$ It is responsible for voluntary movement of skeletal muscles. ${ }^{13}$ The premotor cortex lies anterior to the precentral gyrus and is responsible for the planning of movement based on sensory information. ${ }^{13}$ Broca's area is located anterior to the inferior premotor cortex and is typically present in the left hemisphere only. ${ }^{13}$ The function of this area is to plan and carry out muscular activities involved in speech. ${ }^{13}$ The frontal eye field is located anterior to the premotor cortex and superior to Broca's area. It is responsible for voluntary movement of the eyes. ${ }^{13}$

The sensory areas are located within the parietal, temporal and occipital lobes. The primary somatosensory cortex is located in the postcentral gyrus of the parietal lobe. Neurons in this area receive sensory information from the skin and proprioceptors in muscles, joints and tendons. ${ }^{13}$ The somatosensory association cortex is posterior to the primary somatosensory cortex. It receives information such as pressure and temperature to identify size and shape of objects being touched. ${ }^{13}$

The visual areas are located within the occipital lobe. ${ }^{13}$ The primary visual cortex receives information from the retina of the eye. ${ }^{13}$ This information is transferred to the visual association area. Information from past experiences allows recognition of an object based on colour, movement and shape. ${ }^{13}$

The auditory areas are located within the temporal lobe near the lateral sulcus. Sound impulses are transferred to the primary auditor cortex and are interpreted as pitch, loudness and location. ${ }^{13}$ These stimuli are then transferred to the auditory association area, which 
perceives the sound as speech, music or noise. Memories of past experiences are used as reference. $^{13}$

The olfactory cortex is also located within the temporal lobe. Sensory receptors in the nasal cavity transmit information to the olfactory cortices, allow the perception of different aromas. $^{13}$

While the cerebellum mainly controls equilibrium, a small portion of the vestibular cortex is located within the insula and parietal cortex. This area is responsible for balance and proprioception. $^{13}$

The insula also contains the gustatory cortex, which is responsible for taste, as well as the visceral sensory area which receives sensory information from internal organs. ${ }^{13}$

There are also many multimodal association areas within the brain. These areas integrate information from multiple areas. The anterior association area, or prefrontal cortex, is located in the frontal lobe. It is a very complicated region and is involved in learning, memory recall and personality. ${ }^{13}$ The posterior association area is located in the temporal, parietal and occipital lobes. Recognition of patterns and faces occurs in this area, as well as the understanding of spoken and written language. ${ }^{13}$ The limbic association area is composed of the cingulate gyrus, the parahippocampal gyrus and the hippocampus. It is responsible for emotional impact of an event. ${ }^{13}$

The white matter of the cerebrum is located internal to the cerebral cortex/gray matter. It contains myelinated fibres which are arranged into large tracts. ${ }^{13}$ Association fibres form connections within a hemisphere. Commissural fibres connect gray areas between hemispheres, allowing the brain to function as a whole. ${ }^{13}$ Projection fibres carry sensory or motor information, and form the connection from the cerebral cortex to the rest of the body. ${ }^{13}$ 
The third layer of the cerebrum is known as the basal nuclei or basal ganglia. This area receives information from the cerebral cortex, subcortical nuclei and other basal nuclei. While not well understood, this area plays a role in movement, cognition and emotion, filtering out inappropriate responses and projecting only pertinent information to the cortex. ${ }^{13}$

The diencephalon is composed of the thalamus, hypothalamus and epithalamus. It is located within the centre of the brain. The thalamus accounts for approximately $80 \%$ of the diencephalon. ${ }^{13}$ It contains many nuclei, which projects information to various parts of the cortex. Sensory information from the body is relayed to the thalamus where it is sorted and sent to the appropriate area of the cortex..$^{13}$

The hypothalamus controls visceral function and homeostasis. It is responsible for control of the autonomic nervous system, physical response to emotions such as increased heart rate, endocrine system function and regulation of body temperature, food and water intake and sleep-wake cycles. ${ }^{13}$

The epithalamus contains the pineal gland, which secretes melatonin. This area aids in regulation of the sleep-wake cycle. ${ }^{13}$

The cerebellum is known as the "little brain". It sits posterior to the pons and medulla and inferior to the cerebrum, and accounts for $11 \%$ of total brain mass. It can be divided into two lateral hemispheres by the vermis. ${ }^{11}$ The cerebellum controls synergistic muscle action ${ }^{12}$ by providing appropriate patterns of skeletal muscle contraction. ${ }^{13}$

The brainstem is composed of three parts: the midbrain, pons and medulla oblongata. ${ }^{13}$ This area makes up approximately $2.5 \%$ of total brain mass. Collectively, the brainstem forms the connection between the brain and spinal cord, and is responsible for many involuntary actions required for survival. ${ }^{13}$ 
The midbrain is the most superior portion of the brainstem. It contains pathways of the autonomic nervous system and controls the "fight or flight" response. ${ }^{13}$ It is also responsible for control of the occulomotor and trochlear cranial nerves. ${ }^{13}$

The pons is the middle section of the brain stem and contains many conduction tractions. ${ }^{13}$ It is associated with the trigeminal, abducens and facial cranial nerves. ${ }^{13}$

The medulla oblongata is the most inferior portion of the brainstem. This section of the brainstem plays an important role in homeostasis. It controls the cardiovascular and respiratory centres and is necessary for survival. ${ }^{13}$

The brain is surrounded by the cranial meninges, which lie between the cerebrum and the internal surface of the skull. The function of the meninges is to protect the brain and house arteries and veins. ${ }^{11}$ There are three meningeal layers: the dura mater, arachnoid mater, and pia mater. $^{11}$

The dura mater consists of a tough fibrous substance. It is composed of two layers. The periosteal layer is continuous with the cranium. The meningeal layer creates dural folds such as the cerebral falx and the cerebellar tentorium, which separate the different hemispheres and lobes of the brain. ${ }^{11}$ Located beneath the dura mater is the subdural space. This is a small cavity which contains a thin layer of fluid. ${ }^{13}$

The arachnoid mater is the middle meningeal layer. It is composed of a thin elastic connective tissue. ${ }^{13}$ The subarachnoid space lies between the arachnoid and the pia mater. Cerebrospinal fluid runs through this space, providing protection for the brain. ${ }^{11}$

The pia mater is the innermost meningeal layer. It is composed of the same thin connective tissue as the arachnoid mater. ${ }^{13}$ The pia mater adheres to the surface of the brain, following every fissure. It is highly vascularized..$^{11,12,13}$ 


\section{Neurovascular Structures of the Brain}

Cerebrospinal fluid surrounds the structures of the central nervous system and provides a protective cushioning. ${ }^{13}$ It effectively causes the brain to "float", reducing its weight by $97 \%$. Without the cerebrospinal fluid, the brain would be crushed under its own weight. Cerebrospinal fluid is important in protecting the brain and spinal cord from trauma, as well as providing nourishment. ${ }^{13}$ It is composed of a watery substance and many ions such as sodium, chlorine, hydrogen, calcium and potassium. It is similar to blood plasma, but contains different ion concentrations. ${ }^{13}$ Cerebrospinal fluid is produced by the choroid plexuses located within the ventricles. $^{13}$

A continuous network of ventricles exists throughout the brain. These large cavities contain cerebrospinal fluid. They are lined with ependymal cells, which aid in the movement of cerebrospinal fluid throughout the brain and spinal cord. ${ }^{13} \mathrm{~A}$ large lateral ventricle is located within each cerebral hemisphere. The lateral ventricles connected to the third ventricle in the diencephalon via the interventricular foramen. ${ }^{13}$ The third ventricle connects to the fourth ventricle through the cerebral aqueduct. The fourth ventricle is located in the hindbrain posterior to the pons and medulla. ${ }^{13}$ The fourth ventricle is connected to the subarachnoid space through three openings: two lateral apertures and the median aperture. ${ }^{13}$ From the fourth ventricle, the cerebrospinal fluid travels into the central canal of the spinal cord. ${ }^{13}$

There are twelve pairs of cranial nerves associated with the brain. With the exception of the vagus nerve, the cranial nerves control head and facial movements. Cranial nerves I and II are associated with the forebrain. Cranial nerves III - XII are associated with the brainstem. ${ }^{13}$ Due to the direct association with the brain, cranial nerve testing has been an important factor in concussion diagnosis. 
Cranial nerve I, the olfactory nerve, sends sensory information for smell to the primary olfactory cortex. Cranial nerve II, the optic nerve, transmits information from the retina to the optic chiasma. From here, sensory information for vision is carried to the visual cortex. Cranial nerve III, the occulomotor nerve, controls pupil function as well as the extrinsic eye muscles in conjunction with cranial nerve IV - the trochlear nerve. Cranial nerve V, the trigeminal nerve, transmits sensory information from the superior portion of the face, the nasal cavity and the tongue (not including the taste buds). Cranial nerve VI, the abducens nerve, controls the lateral extrinsic muscle of the eye. Cranial nerve VII, the facial nerve, is the main motor nerve of the face, controlling several facial muscles. Cranial nerve VIII, the vestibulocochlear nerve, transmits sensory information from the inner ear and is responsible for equilibrium and balance. Cranial nerve IX, the glossopharyngeal nerve, innervates the tongue and pharynx and is involved in the swallowing mechanism as well as taste and sensory information from the mouth. Cranial nerve $X$, the vagus nerve, aids in the regulation of various organs involved autonomic activity. Cranial nerve $\mathrm{XI}$, the accessory nerve, controls motor function of the trapezius and sternocleidomastoid muscles. Cranial nerve XII, the hypoglossal nerve, controls tongue movement involved in chewing, swallowing and speech. ${ }^{13}$

Blood supply to the brain comes from two major arteries. These include the common carotid arteries, as well as the vertebral arteries which branch off the subclavian artery. ${ }^{13}$ The left common carotid artery originates from the brachiocephalic trunk, while the left branches off the aortic arch. ${ }^{13}$ At the superior border of the larynx, each common carotid arteries divides into an internal and external carotid artery. ${ }^{13}$ The external arteries supply many structures of the head and face such as the tongue, anterior facial muscles and larynx. ${ }^{13}$ The internal arteries supply $80 \%$ of the cerebrum. ${ }^{13}$ They enter the brain through the carotid canals of the temporal 
bone, where they branch off into the anterior and middle cerebral arteries. ${ }^{13}$ The anterior cerebral arteries supply the medial portion of the frontal and parietal lobes. The anterior communicating artery connects the two anterior cerebral arteries. ${ }^{13}$ The middle cerebral arteries supply the lateral portions of the temporal, parietal and frontal lobes. ${ }^{13}$

The vertebral arteries enter the skull through the foramen magnum. Once inside the cranium, the left and right vertebral arteries joint to form the basilar artery which supplies blood to the cerebellum and pons. ${ }^{13}$ At the junction between the pons and the midbrain, the basilar artery divides to form the posterior cerebral arteries, supplying the occipital lobes as well as the inferior portion of the temporal lobes. ${ }^{13}$ Two posterior communicating arteries connect the posterior cerebral arteries to the middle cerebral arteries.

All blood supply from the common carotid and vertebral arteries are linked, forming a connection of blood vessels called the Circle of Willis. This structure aids in the maintenance of blood pressure in the brain, and allows alternate pathways for blood supply, decreasing the effects of an occluded artery. ${ }^{13}$

The blood brain barrier is a protective mechanism that maintains a constant environment in the brain. ${ }^{13}$ Capillaries within the brain are the least permeable capillaries in the body, requiring substances to pass through three layers before reaching the brain tissue. This mechanism allows nutrients to pass through and prevents wastes from entering the brain. ${ }^{13}$

\section{Definition of a Concussion}

Head injuries are typically classified into two types. The first, a focal injury or posttraumatic intracranial mass lesion, includes injuries such as subdural and epidural hematomas, cerebral contusions and intracerebral hemorrhages and hematomas. These types 
of injuries include symptoms such as loss of consciousness, cranial nerve deficits, mental status deterioration, and are serious medical emergencies.

The second type of head injury is known as a diffuse brain injury. These injuries are typically caused by an acceleration or deceleration mechanism, either in a linear or rotational plane, or a combination of both. A review on high school and collegiate football players found that concussions were caused by a linear acceleration of $74.0 \mathrm{~g}-146.0 \mathrm{~g}$ (where $\mathrm{g}$ is the force of gravity) and an angular acceleration of 5,582.6 rads $/ \mathrm{s}^{2}-9,515.6 \mathrm{rads} / \mathrm{s}^{2} .{ }^{14}$ When a large force is applied to the head, the brain moves around hitting the inside of the skull, resulting in tissue damage. Concussions are typically classified as a mild diffuse injury, also known as a mild traumatic brain injury (MTBI). ${ }^{6}$ Each year, between $1.4-3.8$ million concussions occur in the United States, many of which are sport-related. ${ }^{15,16}$

The word "concussion" is derived from the Latin word "concussus", meaning to shake violently. In 1966, the Congress of Neurological Surgeons defined a concussion as "a clinical syndrome characterized by immediate and transient impairment of neural functions, such as alterations of consciousness, disturbance of vision, equilibrium, etc., due to mechanical forces." ${ }^{6}$ In more recent years, however, the general consensus is that this definition is too vague and does not include important factors of a concussion such as describing common symptoms. While a current standard definition does not exist, it is generally accepted that a concussion involves several main concepts. Concussions are caused by either a direct blow to the head, or to another part of the body with force being transmitted to the head. This may cause a temporary neurologic deficit, and will typically result in a variety of symptoms. Neurologic changes will typically be functional only, and neurological images will appear normal. ${ }^{6}$ 
A concussion can occur as a result of two mechanisms. The first, a coup injury, occurs when a blow to the head at rest causes damage on the ipsilateral side of the brain.

The second, a contracoup injury, occurs when the head is in motion and comes in contact with a stationary object. The brain shifts to the opposite side of the skull, causing a contralateral injury. Currently, there is no evidence to suggest that one mechanism is more severe than the other, and both have been shown to result in similar symptoms. ${ }^{6}$

Each incidence of a concussion is unique. This has caused many problems with respect to creating a universally accepted grading scale. There are three different approaches that have been created to classify and grade concussions. The first is based on the presence or absence of loss of consciousness and symptom resolution within the first 15 minutes after injury. It uses the American Academy of Neurology Concussion Grading Scale. ${ }^{6}$ A Grade 1 or mild concussion includes no loss of consciousness and symptom resolution within 15 minutes. A Grade 2 or moderate concussion occurs when there is no loss of consciousness but symptoms continue for 15 minutes or longer. Any loss of consciousness is classified as a Grade 3 or severe concussion. ${ }^{6}$

A second method uses the Cantu Evidence-Based Grading System for Concussion scale. ${ }^{17}$ In this approach, the concussion is graded after complete symptom resolution. A Grade 1 or mild concussion is defined as no loss of consciousness, with posttraumatic amnesia either absent or lasting less than 30 minutes, and all other symptoms resolved within 24 hours post injury. A Grade 2 or moderate concussion occurs when there is loss of consciousness for less than one minute, when posttraumatic amnesia persists for longer than 30 minutes, or when symptoms last for 1-7 days. A Grade 3 or severe concussion has a loss of consciousness greater than one minute, posttraumatic amnesia for more than 24 hours, or persistent symptoms for longer than one week. ${ }^{17}$ 
Recent research has shown that severity of a concussion is not dependant upon loss of consciousness or presence of amnesia. These findings make both the American Academy of Neurology and the Cantu concussion grading scales ineffective, and concussion diagnosis has moved away from the use of these two scales.

The third approach does not include a grading scale. It uses symptom checklists, neuropsychological tests and postural evaluations to determine severity. This approach is popular because it does not require the medical professional to categorize a concussion, and allows each case to be treated individually. Once the individual is asymptomatic, a step-by-step progression is used to gradually return to activity. This approach was first introduced at the 2001 Vienna Conference on Concussion in Sport, ${ }^{1}$ and is the accepted method for classifying concussions.

\section{Pathophysiology of a Concussion}

When a concussion is sustained, there are changes in normal brain function due to neuronal, chemical and neuroelectrical deficits. ${ }^{17}$ Structural damage may also occur and result in the loss of brain cells. ${ }^{17}$ Surviving brain cells remain in a vulnerable state for several days postinjury, and are susceptible to minor changes that would not normally have an effect on a healthy brain. This can include changes in cerebral blood flow, intracranial pressure and anoxia. ${ }^{17}$ Animal studies have shown that a slight decrease in cerebral blow flow during this period of vulnerability results in the loss of additional brain cells. ${ }^{18-22}$

While this process is still not fully understood, the vulnerability of healthy brain cells may be due to a neurometabolic cascade. Upon impact, the brain is forced to accelerate, resulting in stretching and shearing of neurons. ${ }^{23,24,25}$ This causes defects in the neuronal membranes, allowing ions to flow freely in and out of the nerve cells, resulting in 
depolarization. ${ }^{26}$ As a result of this depolarization, the excitatory amino acid, glutamate, is released, promoting potassium release and further depolarization caused by the activation of $\mathrm{N}$ methyl-d-aspartate (NMDA) receptors. ${ }^{26}$ This portion of the cascade is excitatory, causing the immediate signs often seen with concussion such as confusion, seizure, convulsions and problems with vision and balance. ${ }^{23-25,27}$

Activation of the NMDA receptors also causes a large influx of calcium ions into the neuron. This extra calcium overloads intracellular mitochondria and results in a decrease in energy production. ${ }^{26}$ With this mitochondrial dysfunction, the neuron is unable to produce adequate amounts of ATP, and may begin producing reactive oxygen species (ROS), which may have damaging effects if the cell is unable to detoxify it. ${ }^{24,25,28}$ The extra intracellular calcium may also cause damage to microtubules and neurofilaments in the axon, resulting in a disruption of neural connectivity. ${ }^{25}$

After this initial excitatory stage, there is a global suppression of neurons. This period is known as "spreading depression", and causes other concussive signs and symptoms to appear such as cognitive deficits, amnesia and fatigue..$^{23,25,27}$

The result of this neurometabolic cascade is an imbalance between intracellular and extracellular ions. This process is reversible, but requires maximal function of the $\mathrm{Na}+/ \mathrm{K}+$ pump to return to normal cell homeostasis. ${ }^{26}$ The issue that arises is that this pump is heavily dependent on ATP for energy. Due to mitochondrial dysfunction, the cell must rely on lessefficient means of energy production such as anaerobic glycolysis. ${ }^{26}$ Energy production is further inhibited by a decrease in cerebral blood flow, resulting in a decreased availability of glucose. ${ }^{25}$ This decrease in blood flow is thought to occur as a way to prevent massive cerebral swelling. ${ }^{292726,22,21}$ 
Following impact to the brain, there is a deficit in cerebral energy. There is a much greater demand for ATP production than the brain is capable of producing. This state has been shown to last for up to two weeks in animal models, and may be extended longer in humans. ${ }^{30}$

Many signs and symptoms seen after concussion may occur as a result of damage to certain areas of the brain. Posttraumatic amnesia may be seen with damage to the cerebral cortex, subcortical projections, hippocampus and diencephalon. ${ }^{31}$ Lesions of the frontal lobe may cause behavioural changes such as irritability, aggressiveness and loss of judgement. ${ }^{32}$

\section{Complications Following a Concussion}

Epidural and subdural hematomas can lead to intracranial bleeding. These conditions can accompany a concussion. Intracranial bleeding is a serious medical emergency requiring immediate care. A CT Scan is required to determine the presence of either type of bleed. Epidural hematomas are caused by a tear to the meningeal arteries following an impact. ${ }^{12} \mathrm{An}$ athlete or patient with an epidural hematoma will typically present with a history of head injury accompanied by a short loss of consciousness. ${ }^{33}$ After consciousness is regained, a lucid period typically follows before progressing rapidly to a second period of unconsciousness. Symptoms to look for during the lucid period include ipsilateral pupil dilation and contralateral side paralysis. ${ }^{33}$ These symptoms and change in level of consciousness will typically occur within a short span of time due to the arterial nature of the bleed. Subdural hematomas occur when the veins between the dura mater and the brain are torn by shear force during acceleration and deceleration of the brain. ${ }^{12}$ Symptoms of a subdural bleed will typically progress much slower than an epidural hematoma. An acute subdural hematoma will show symptoms within 24 hours, while a subacute subdural hematoma may not show symptoms for $2-10$ days post-injury. ${ }^{33}$ 
Common symptoms of a subdural hematoma include drowsiness, confusion, apathy and a decreasing level of consciousness. ${ }^{33}$

Post-concussion syndrome is an exacerbation of the psychological and cognitive symptoms of concussion prolonged during an extended period of time with an unknown underlying mechanism. ${ }^{34}$ The clinical criteria for post-concussion syndrome as defined by the World Health Organization as having three or more of the following symptoms: headache, dizziness, fatigue, irritability, insomnia, concentration difficulty or memory difficulty. Typically these symptoms arise within four weeks from the original injury. ${ }^{27}$ Although little is known about this condition, it is thought to be caused by psychological distress that develops when an individual has an extended recovery time. ${ }^{35}$ Those who have a history of prior concussion or psychological disorder are at a higher risk for developing post-concussion syndrome. Postconcussion syndrome is often misdiagnosed due to its similarity with other psychological disorders such as anxiety and depression. ${ }^{34}$

Second Impact Syndrome occurs when an individual suffers a second concussion before a previous concussion is fully resolved. ${ }^{36}$ While this condition is not well understood, the theory behind it states that the initial environment caused by the first concussion is exacerbated with a second impact. The brain is not capable of handling a second neurometabolic cascade in its damaged state, resulting in a loss of cerebral blood flow regulation, swelling and eventually death. ${ }^{24,25}$ Cerebral swelling causes an increase in intracranial pressure, leading to brainstem herniation. ${ }^{37}$ Damage to the brainstem affects the breathing and cardiac control centers, which eventually leads to death. Reports have shown that death from second impact syndrome occurs within 2-5 minutes after impact. ${ }^{38}$ If a patient is suspected to have second impact syndrome, they should be immediately stabilized at the level of the cervical spine. Special attention should 
be paid to airway maintenance and the athlete should be transported for further care as soon as possible.

Chronic Traumatic Encephalopathy (CTE) is a neurodegenerative disorder caused by repeated head trauma. ${ }^{39}$ It has been studied extensively in boxers, and is now being seen in athletes from other contact sports such as football and soccer. ${ }^{40}$ Symptoms typically include neurological, cognitive, psychiatric and behavioural changes. ${ }^{41}$ In the early stages of this disease, attention, concentration and memory are affected. Disorientation and confusion are also common. As the brain progressively deteriorates, lack of insight, poor judgment, and dementia are seen. In severe cases, muscular movements become slowed, gait and speech are affected, and tremors begin. ${ }^{42}$ The development of Parkinsonism may also be seen during the second and third stages. ${ }^{43}$ Symptoms typically develop later in life, and often years after the completion of the athlete's career. ${ }^{40}$ Common pathology seen with CTE includes a reduction in brain weight, enlargement of the lateral and third ventricles, thinning of the corpus callosum, cavum septum pellucidum with fenestrations, and scarring and neuronal loss of the cerebellar tonsils. ${ }^{43}$ The reduction is brain weight is typically due to atrophy of the frontal, temporal and parietal lobes. ${ }^{43}$ Neurofibrillary tangles and tau protein formation are two common biological abnormalities seen with CTE. In a normal brain, tau proteins promote microtubule assembly. ${ }^{39}$ In a CTE brain, these proteins are phosphorylated, causing a disassembly of microtubules leading to impaired axonal transport and synaptic function. ${ }^{44}$ Currently there are no accepted diagnostic criteria for CTE.

\section{Evaluation of a Concussion}

There are many tools available to aid in the assessment of a concussion. These tools typically identify the presence of symptoms, as well as cognitive and postural or balance deficits. 
Before testing for a concussion, it is essential to rule out more severe injuries such as a skull fracture or intracranial bleed. The cranial and facial bones should be palpated to check for tenderness, deformation and lacerations. Although neuroimaging typically does not show signs of a concussion, it is necessary if a more severe injury is suspected. The Canadian CT Head Rules have been shown to have $100 \%$ sensitivity and $76.3 \%$ specificity in determining whether a head injury patient requires neurosurgery. ${ }^{45}$ The Canadian CT Head Rule states that a patient with minor head injury and a Glasgow Coma Scale score of 13-15 who experienced loss of consciousness, amnesia or confusion is at high risk for neurosurgical intervention if they experience any of the following: a Glasgow Coma Scale score of $<152$ hours post-injury, a suspected skull fracture, any sign of basal skull fracture (raccoon eye's, Battle's sign, etc.), two or more episodes of vomiting, or are 65 years or older. Patients are at a medium risk for brain injury detection by a CT scan if they have experienced retrograde amnesia for 30 minutes or more leading up to impact, or have a dangerous mechanism of injury such as a fall from greater than 3 feet or a serious motor vehicle accident. ${ }^{26}$ If the patient or athlete presents with any of these findings, they should be immediately transported for further neuroimaging.

Tests for concussions are used most effectively when a pre-season baseline is established. This allows the identification of a "normal" state for each individual. If a concussion is sustained, testing should be done immediately post-injury, throughout the recovery process, and before the athlete returns to play. Comparisons made against the baseline scores can show progress as the athlete recovers. While normative data for some tests does exist, the most accurate comparisons are made against baseline values.

It is also important to collect previous injury history on each athlete. With regards to previous concussions, important information should include: number of previous concussions, 
mechanism of injury, previous symptoms experienced, recovery time, and previous treatment received. This can help to identify any patterns related to each individual. ${ }^{6}$

A symptom checklist is one of the simplest tools to evaluate a concussion. Different models include a "yes/no" checklist, or a Likert scale in which the individual is asked to rate each symptom based on severity. ${ }^{6}$ The symptom checklist should be administered daily throughout the recovery process, as well as during the return to play program. Caution should be used with this tool, as many athletes will underreport symptoms in order to speed up the return to play process. ${ }^{5}$ A symptom checklist should never be used in isolation or as a definitive factor in decision-making.

The Standardized Assessment of Concussion (SAC) ${ }^{46}$ test is most commonly used to evaluate cognitive deficits and mental status in a concussed athlete. The SAC is broken down into three categories, which evaluate orientation, immediate and delayed memory, and concentration. Scores are summed for a total of 30 points. ${ }^{46}$ To test orientation, the athlete is asked five questions about the date and time, for a total of five points. To test immediate memory, the athlete is read a series of five words at a speed of one word per second, then asked to repeat the words back in any order. This is repeated three times, for a total of 15 points. At a later time, the athlete is asked to repeat the word list back once, without it being read again. This tests delayed memory recall and is worth an additional five points. The final portion of the SAC evaluates concentration with two tests. First, the athlete is read a series of 3, 4, 5 and 6 numbers, and asked to repeat them back in reverse order. If they are unable to complete a trial after two attempts, they will receive a score of zero for each longer trial. This portion is worth four points. The final test of the concentration section asks the athlete to repeat the months in reverse order starting with December. ${ }^{47}$ All points are added together, 
with a lower score indicating a higher degree of cognitive deficit. ${ }^{6}$ The SAC has shown a high level of reliability and validity in determining changes in mental status post-concussion. A drop of one point or more when compared to a baseline score has been associated with $94 \%$ sensitivity and $76 \%$ specificity of detecting cognitive deficits. ${ }^{48}$ Studies of high school athletes have shown baseline SAC scores of between $25.19(+3.10)$ to $26.9(+2.3) .{ }^{49,50}$ The Balance Error Scoring System (BESS) ${ }^{51}$ is used to test the effects of concussion on posture and balance. The athlete is instructed to stand still with their eyes closed for 20 seconds. The test includes three positions: a double leg stance; a single leg stance on the nondominant foot; and a tandem stance where the athlete stands heel to toe with the dominant leg in front. Each position is completed on a flat and foam surface and any deviations from starting position are recorded. A deviation is classified as any movement away from the starting position, and can include errors such as: removing hands from hips, opening eyes, stumbling, touching foot to the ground, moving the hip into greater than 30 degrees of abduction, or remaining out of the starting position for longer than 5 seconds. ${ }^{47}$ Each testing position is scored out of 10 points, with one point lost for each deviation from the starting position. In addition, a subject who cannot maintain the testing position for 5 seconds at the start receives a score of zero. ${ }^{47}$ In a study of high school athletes, the average baseline BESS score was 25.82 out of a total of 30 points. $^{50}$

The Sport Concussion Assessment Tool 2 (SCAT2) is a helpful tool for sideline assessment. This test incorporates a symptom checklist, Glasgow Coma Scale, questions of orientation (Maddock's Scale), as well as SAC, BESS and coordination tests. ${ }^{47}$ The total score for the SCAT2 is 100 points. Studies of high school athletes have shown that the average baseline score falls between $87-92.3 .^{49,50}$ 
Neuropsychological testing is also an important component of a concussion evaluation. This type of testing evaluates constructs such as reaction time, information processing, problem solving, attention and memory. ${ }^{6}$ Traditionally, these tests were administered in a paper and pencil format by neuropsychologists. Recently, many computer-based tests have become available. Examples of such include the Automated Neuropsychological Assessment Metrics (ANAM), CogSport, Concussion Resolution Index, and Immediate Postconcussion Assessment and Cognitive Testing (ImPACT). ${ }^{6}$ Computer-based tests have many advantages, including ease of administration and multiple variations to prevent practice effects. ${ }^{6}$ Access to this type of testing has become important in the return to play decision-making process. Recent research has shown that athletes may continue to exhibit neurocognitive deficits even though symptoms has resolved. ${ }^{5,52}$ One study found that $38 \%$ of participants tested using the ImPACT system showed cognitive deficits even after reporting being asymptomatic. ${ }^{5}$

\section{Concussion Management}

Once an individual has been diagnosed with a concussion, there are many steps that need to be taken to promote a successful recovery. The Third International Conference on Concussions in Sport states that an athlete who sustains a concussion is not to return to play the same day. ${ }^{3}$ The athlete should refrain from any activity that exacerbates concussion symptoms, and should be frequently evaluated by a medical professional for changes in symptoms, cognition and balance. If symptoms increase at any time, the athlete should be referred to a physician for possible neuroimaging. ${ }^{6}$

For the first 24 hours after injury, a friend, parent, or roommate who can watch for worsening symptoms or a decline in mental status should monitor the athlete. Such instances may be indicative of an intracranial bleed or other serious medical conditions. ${ }^{33}$ 
Medications containing aspirin or non-steroidal anti-inflammatory drugs (NSAIDs) should be avoided. These have been shown to decrease platelet function and increase the possibility of intracranial bleeding, in addition to diminishing existing symptoms. ${ }^{6}$ Alcohol and narcotics should also be avoided, as they have an effect on nervous system function, and can also mask symptom changes. ${ }^{6}$

Originally, it was thought that an athlete should be awakened every 3-4 hours at night to determine if any symptom changes had occurred. However, recent evidence from research has shown that this may be unnecessary and may have an adverse reaction on symptoms due to a disturbance of the sleep cycle. ${ }^{6}$

As symptoms begin to decrease, the individual should resume activities of daily living such as work, school and driving, but should refrain from any activities that put them at risk of further injury or exacerbation of symptoms until completely symptom-free. The individual should make every attempt to achieve "cognitive rest", meaning that activities requiring intense concentration should be avoided. This includes activities involving computer usage, playing video games and watching television. These activities may cause an increase in symptoms. ${ }^{6}$

Strenuous exercise should be avoided, as it has been shown to prolong symptoms.

Exercise varies the amount of glucose uptake in the brain. ${ }^{53}$ As previously discussed, the concussed brain is already lacking glucose for energy production. Exercise may worsen this condition, causing prolonged symptom resolution.

\section{Return to Play}

After 24 hours of being symptom-free, the athlete may begin a stepwise return to play program. This protocol was developed at the First International Conference on Concussion in Sport in 2001, and has been the accepted standard since. ${ }^{1,3}$ This protocol is composed of six 
steps. Each step should be completed a minimum of 24 hours after the previous stage. If the athlete develops symptoms at any stage, they are to repeat the previous stage after 24 hours and wait an additional 24 hours before attempting to progress. The entire protocol takes a minimum of six days to complete if the athlete is able to progress through each stage asymptomatically.

Stage one is a period of physical and mental rest. ${ }^{1}$ The athlete should refrain from exercise and any cognitively challenging activities, such as studying, watching tv and playing video games. Stage two allows the athlete to begin light aerobic exercise such as cycling or swimming. ${ }^{1}$ In stage 3 , sport-specific aerobic exercises such as skating and running are added. ${ }^{1}$ The athlete may return to sport-specific activity drills in stage 4 , but should avoid any contact. ${ }^{1}$ Types of activities permitted in stage 4 include dribbling, shooting and catching. Before progressing to stage 5 , the athlete should be cleared for participation by a medical professional. They may then participate in a full-contact practice with teammates. The final stage allows the athlete to return to competition. ${ }^{1}$ It has been suggested that the athlete be challenged with a "cognitive training" period between stages one and two. This may include light cognitive activities such as reading. ${ }^{26}$

The athletic trainer plays a crucial role in the return to play process. Often, the athletic trainer is responsible for monitoring the athlete's symptoms and performance throughout each stage. The decision to return an athlete to play typically falls to the team physician or sports medicine team, although the athletic trainer may be responsible for this decision if there is not a physician present. 


\section{REFERENCES}

1. Aubry M, Cantu R, Dvorak J, et al. Summary and agreement statement of the first International Conference on Concussion in Sport, Vienna 2001. Br J Sports Med. 2002;36(1):6-7. doi:10.1136/bjsm.36.1.6.

2. McCrory $P$, Johnston $K$, Meeuwisse $W$, et al. Summary and agreement statement of the 2 nd International Conference on Concussion in Sport, Prague 2004. Br J Sports Med. 2005;39(4):196-204. doi:10.1136/bjsm.2005.018614.

3. Cantu RC. CONSENSUS STATEMENT ON CONCUSSION IN SPORT - THE 3RD INTERNATIONAL CONFERENCE ON CONCUSSION, ZURICH, NOVEMBER 2008. Neurosurgery. 2009;64(5):786-787. doi:10.1227/01.NEU.0000348539.13887.D3.

4. Lovell M, Collins M, Bradley J. Return to play following sports-related concussion. Clin Sports Med. 2004;23:421-441.

5. Broglio SP, Macciocchi SN, Ferrara MS. Neurocognitive Performance of Concussed Athletes When Symptom Free. J Athl Train. 2007;42(4):504-508.

6. Guskiewicz KM, Bruce SL, Cantu RC, et al. National Athletic Trainers' Association Position Statement: Management of Sport-Related Concussion. J AthI Train. 2004;39(3):280-297.

7. Notebaert AJ, Guskiewicz KM. Current Trends in Athletic Training Practice for Concussion Assessment and Management. J Athl Train. 2005;40(4):320-325.

8. Ferrara MS, McCrea M, Peterson CL, Guskiewicz KM. A Survey of Practice Patterns in Concussion Assessment and Management. J Ath/ Train. 2001;36(2):145-149.

9. Lynall RC, Laudner KG, Mihalik JP, Stanek, Justin M. Concussion-Assessment and Management Techniques Used by Athletic Trainers. J AthI Train. 2013;48(8):844-850.

10. Gray H, Lewis WH. Anatomy of the Human Body. 20th ed. Philadelphia: Lea and Febiger; 1918. Available at: http://www.bartleby.com/107/.

11. Moore KL, Dalley AF. Clinically Oriented Anatomy. 5th ed. United States of America: Lippincott Williams \& Wilkins; 2006. 
12. Prentice W. Arnheim's Principles of Athletic Training: A Compentency-Based Approach. 13th ed. Versailles: McGraw-Hill; 2009.

13. Marieb E, Hoehn K. Human Anatomy \& Physiology. 9th ed. United States of America: Pearson Education Inc; 2013.

14. Broglio SP, Surma T, Ashton-Miller JA. High School and Collegiate Football Athlete Concussions: A Biomechanical Review. Ann Biomed Eng. 2012;40(1):37-46. doi:10.1007/s10439-011-0396-0.

15. CDC - Statistics - Traumatic Brain Injury - Injury Center. Available at: http://www.cdc.gov/traumaticbraininjury/statistics.html. Accessed September 11, 2013.

16. Langlois J, Rutland-Brown W, Wald M. The Epidemiology and Impact of Traumatic Brain Injury: A Bri... : The Journal of Head Trauma Rehabilitation. Available at: http://journals.Iww.com/headtraumarehab/Fulltext/2006/09000/The_Epidemiology_an d_Impact_of_Traumatic_Brain.1.aspx. Accessed September 11, 2013.

17. Cantu RC. Posttraumatic Retrograde and Anterograde Amnesia: Pathophysiology and Implications in Grading and Safe Return to Play. J Athl Train. 2001;36(3):244-248.

18. Jenkins LW, Marmarou A, Lewelt W, Becker DP. Increased Vulnerability of the Traumatized Brain to Early Ischemia. In: Baethmann A, Go KG, Unterberg A, eds. Mechanisms of Secondary Brain Damage. NATO ASI Series. Springer US; 1986:273-282. Available at: http://link.springer.com/chapter/10.1007/978-1-4684-5203-7_21. Accessed July 3, 2013.

19. Jenkins LW, Moszynski K, Lyeth BG, et al. Increased vulnerability of the midly traumatized rat brain to cerebral ischemia: the use of controlled secondary ischemia as a research tool to identify common or different mechanisms contributing to mechanical and ischemic brain injury. Brain Res. 1989;477(1-2):211-224. doi:10.1016/0006-8993(89)91409-1.

20. Lee S, Lifshitz J, Hovda D, Becker D. Focal cortical-impact injury produces immediate and persistent deficits in metabolic autoregulation. J Cereb Flow Metab. 1955;15(1):22.

21. Lifshitz J, Pinanong P, Le H, Lee S, Hovda D, Becker D. Regional uncoupling of cerebral blood flow and metabolism in degenerating cortical areas following a lateral cortical contusion. J Neurotrauma. 1995;12:129.

22. Ph.D RLS, Hovda DA, Adelson PD, Benzel EC, Becker DP. Metabolic Changes Following Cortical Contusion: Relationships to Edema and Morphological Changes. In: M.D UI, M.D AB, M.D K-AH, et al., eds. Brain Edema IX. Acta Neurochirurgica. Springer Vienna; 1994:446-448. Available at: http://link.springer.com/chapter/10.1007/978-3-70919334-1_122. Accessed July 3, 2013. 
23. Khurana VG, Kaye AH. An overview of concussion in sport. J Clin Neurosci. 2012;19(1):1-11. doi:10.1016/j.jocn.2011.08.002.

24. Signoretti S, Lazzarino G, Tavazzi $\mathrm{B}$, Vagnozzi R. The Pathophysiology of Concussion. PM\&R. 2011;3(10, Supplement 2):S359-S368. doi:10.1016/j.pmrj.2011.07.018.

25. Giza CC, Hovda DA. The Neurometabolic Cascade of Concussion. J Athl Train. 2001;36(3):228235.

26. Marshall CM. Sports-related concussion: A narrative review of the literature. $J$ Can Chiropr Assoc. 2012;56(4):299-310.

27. Ropper AH, Gorson KC. Concussion. N Engl J Med. 2007;356(2):166-172.

28. Vagnozzi R, Marmarou A, Tavazzi B, et al. Changes of Cerebral Energy Metabolism and Lipid Peroxidation in Rats Leading to Mitochondrial Dysfunction After Diffuse Brain Injury. J Neurotrauma. 1999;16(10):903-913. doi:10.1089/neu.1999.16.903.

29. Brain Tissue pH in Severely Head-injured Patients: A Report... : Neurosurgery. Available at: http://journals.Iww.com/neurosurgery/Fulltext/1987/02000/Brain_Tissue_pH_in_Sever ely_Head_injured_Patients_.17.aspx. Accessed July 29, 2013.

30. Hovda D, Prins M, Becker D. Neurobiology of concussion. In: Sports-related concussion. Quality Medical Publishing; 1999:12-51.

31. Adams R, Victor M, Ropper A. Principles of Neurology. 6th ed. New York, NY: McGraw-Hill; 1997.

32. Stuss DT, Shallice T, Alexander MP, Picton TW. A Multidisciplinary Approach to Anterior Attentional Functionsa. Ann N Y Acad Sci. 1995;769(1):191-212. doi:10.1111/j.17496632.1995.tb38140.x.

33. Porth CM. Essentials of Pathophysiology: Concepts of Altered Health States. 2nd ed. United States of America: Lippincott Williams \& Wilkins; 2007.

34. Ryan LM, Warden DL. Post concussion syndrome. Int Rev Psychiatry. 2003;15(4):310-316. doi:10.1080/09540260310001606692.

35. Lishman WA. Physiogenesis and psychogenesis in the "post-concussional syndrome". Br J Psychiatry. 1988;153(4):460-469. doi:10.1192/bjp.153.4.460.

36. Cantu RC. SECOND-IMPACT SYNDROME. Clin Sports Med. 1998;17(1):37-44. doi:10.1016/S0278-5919(05)70059-4. 
37. McCrory P, Davis G, Makdissi M. Second Impact Syndrome or Cerebral Swelling after Sporting Head Injury. Curr Sports Med Rep. 2012;11(1):21-23.

38. Bey T, Ostick B. Second Impact Syndrome. West J Emerg Med. 2009;10(1):6-10.

39. Blennow K, Hardy J, Zetterberg H. The Neuropathology and Neurobiology of Traumatic Brain Injury. Neuron. 2012;76(5):886-899. doi:10.1016/j.neuron.2012.11.021.

40. McKee AC, Cantu RC, Nowinski CJ, et al. Chronic Traumatic Encephalopathy in Athletes: Progressive Tauopathy following Repetitive Head Injury. J Neuropathol Exp Neurol. 2009;68(7):709-735. doi:10.1097/NEN.0b013e3181a9d503.

41. Mendez MF. The Neuropsychiatric Aspects of Boxing. Int J Psychiatry Med. 1995;25(3):249262. doi:10.2190/CUMK-THT1-X98M-WB4C.

42. Millspaugh J. Dementia pugilistica. US Nav Med Bull. 1937;35:297-303.

43. Corsellis J a. N, Bruton CJ, Freeman-Browne D. The aftermath of boxing. Psychol Med. 1973;3(03):270-303. doi:10.1017/S0033291700049588.

44. Mandelkow E-M, Mandelkow E. Biochemistry and Cell Biology of Tau Protein in Neurofibrillary Degeneration. Cold Spring Harb Perspect Med. 2012;2(7). doi:10.1101/cshperspect.a006247.

45. Stiell IG, Clement CM, Rowe BH, et al. Comparison of the canadian CT head rule and the new orleans criteria in patients with minor head injury. JAMA. 2005;294(12):1511-1518. doi:10.1001/jama.294.12.1511.

46. McCrea M, Kelly J, Randolph C. The standardized assessment of concussion: Manual for administration, scoring and interpretation. 1997.

47. Sport Concussion Assessment Tool 2 (SCAT2). Available at: http://www.sportconcussionlibrary.com/content/sport-concussion-assessment-tool-2scat2. Accessed June 26, 2013.

48. W B Barr MM. Sensitivity and specificity of standardized neurocognitive testing immediately following sports concussion. J Int Neuropsychol Soc JINS. 2001;7(6):693-702.

49. Jinguji TM, Bompadre V, Harmon KG, et al. Sport Concussion Assessment Tool - 2: Baseline Values for High School Athletes. Br J Sports Med. 2012;46(5):365-370. doi:10.1136/bjsports-2011-090526.

50. McLeod TCV, Bay RC, Lam KC, Chhabra A. Representative Baseline Values on the Sport Concussion Assessment Tool 2 (SCAT2) in Adolescent Athletes Vary by Gender, Grade, 
and Concussion History. Am J Sports Med. 2012;40(4):927-933.

doi:10.1177/0363546511431573.

51. Riemann BL, Guskiewicz KM. Effects of Mild Head Injury on Postural Stability as Measured Through Clinical Balance Testing. J Athl Train. 2000;35(1):19-25.

52. Fazio VC, Lovell MR, Pardini JE, Collins MW. The relation between post concussion symptoms and neurocognitive performance in concussed athletes. NeuroRehabilitation.

2007;22(3):207-216.

53. Majerske CW, Mihalik JP, Ren D, et al. Concussion in Sports: Postconcussive Activity Levels, Symptoms, and Neurocognitive Performance. J Athl Train. 2008;43(3):265-274. 


\section{APPENDIX \\ SURVEY QUESTIONNAIRE}

\section{Demographic Information}

1. In what year did you become certified as an athletic trainer?

2. How many years have you practiced as an athletic trainer?

3. Which term best describes your current job title?
a. Athletic Trainer
b. Strength and Conditioning Coach
c. Physician Extender
d. Other:

4. Which setting best describes your current job setting?
a. High School
b. College
i. Division:
c. Professional Sports Team
d. Semi-professional Sports Team
e. Private Clinic
f. Hospital
g. Other: 
5. Do you work with athletes? If yes, which sport? Check all that apply.
a. Football
b. Hockey
c. Baseball/Softball
d. Basketball
e. Rugby
f. Volleyball
g. Soccer
h. Other:

6. Is there an assigned physician at your current place of employment? If yes, what type of physician? (General practitioner, neurologist, orthopedic, etc)

\section{Concussion Questions}

7. On average, how many concussions do you see per year at your current place of employment?

8. On a scale of 1-10, how well do you feel you were educated on concussion management during schooling to become an athletic trainer? (1=not educated at all; $10=$ extremely well educated)

9. On a scale of $1-10$, how well do you feel you are currently prepared to manage a

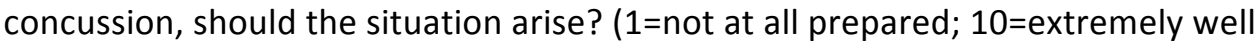
prepared)

10. Have you taken any continuing education seminars/courses related to concussion diagnosis or management? If yes, how many? 
11. Which tools do you use to diagnose concussions at your current place of employment? Check all that apply.
a. SCAT2
b. ImPACT
c. ANAM
d. Symptom checklist
e. BESS
f. SAC
g. Other:

12. Who is responsible for diagnosing a concussion at your current place of employment?
a. Athletic Trainer
b. Team Physician
c. Neurologist
d. Sports Medicine Team
e. Other:

13. Which strategies do you use in the management of concussion? Check all that apply.
a. Bed rest
b. Rest from activity
c. Mental rest
d. Medication
e. Activity modification
f. None
g. Other: 
14. What tests do you use to determine whether or not a player is ready to return to play? Check all that apply.
a. Symptom resolution
b. Return to baseline scores
c. Neuropsychological testing (ImPACT, ANAM)
d. Other:

15. Who is responsible for making return to play decisions at your current place of employment?
a. Athletic Trainer
b. Team Physician
c. Neurologist
d. Sports medicine team
e. Other:

16. Which return to play protocols do you use at your current place of employment? Check all that apply.
a. Complete symptom resolution
b. Step-wise program (Vienna Conference)
c. Neuropsychological tests return to baseline
d. Other:

17. Do you feel that you have ever returned a concussed athlete too soon?
a. Yes
b. No
c. Not applicable 
18. Do you feel that your current place of employment has the appropriate measures in place to properly manage a concussion? (access to testing, adequate training staff/sports medicine team members, etc)
a. Yes
b. No
c. Not applicable

19. On a scale of $1-10$, how involved is your assigned physician throughout the concussion process? (1=not involved; $10=$ involved in every step) Disregard if you do not have an assigned physician.

20. On a scale of $1-10$, how confident are you in the physician's ability to properly manage a concussion? ( $1=$ do not believe physician can manage a concussion; $10=$ physician is extremely proficient at managing concussions) Disregard if you do not have an assigned physician. 